\title{
Bound States in Boson Impurity Models
}

\author{
Tao Shi, Ying-Hai Wu, A. González-Tudela, and J. I. Cirac
}

Max-Planck-Institut für Quantenoptik Hans-Kopfermann-Strasse 1, 85748 Garching, Germany

(Received 23 December 2015; revised manuscript received 24 March 2016; published 25 May 2016)

\begin{abstract}
The formation of bound states involving multiple particles underlies many interesting quantum physical phenomena, such as Efimov physics or superconductivity. In this work, we show the existence of an infinite number of such states for some boson impurity models. They describe free bosons coupled to an impurity and include some of the most representative models in quantum optics. We also propose a family of wave functions to describe the bound states and verify that it accurately characterizes all parameter regimes by comparing its predictions with exact numerical calculations for a one-dimensional tight-binding Hamiltonian. For that model, we also analyze the nature of the bound states by studying the scaling relations of physical quantities, such as the ground-state energy and localization length, and find a nonanalytical behavior as a function of the coupling strength. Finally, we discuss how to test our theoretical predictions in experimental platforms, such as photonic crystal structures and cold atoms in optical lattices.
\end{abstract}

DOI: 10.1103/PhysRevX.6.021027

Subject Areas: Atomic and Molecular Physics

\section{INTRODUCTION}

Boson impurity models, where a two-level system (the impurity) is coupled to a bosonic bath, appear in the description of a variety of physical systems. In particular, they constitute a central paradigm in the field of quantum optics, where the impurity is an emitter (e.g., an atom), and the bosonic bath corresponds to the modes of the electromagnetic field. Despite their simplicity, boson impurity models display a variety of basic phenomena. One of the most intriguing is the existence of a single-excitation bound states (SEBS) for optical emitters interacting with photonic band gap reservoirs [1] (see also Refs. [2,3]), giving rise to interesting phenomena, such as fractional decays or localization phase transitions. In this work, we analyze the existence of multiple-excitation bound states (MEBS) in very generic boson impurity models, including those considered in Refs. [1-3] and other central problems in quantum optics. The existence of bound states lies at the heart of many exotic phenomena, like three-body Efimov states [4], and also very practical ones, like Cooper pairs in superconductivity [5] or polarons [6,7] in electron transport. Furthermore, the interest in MEBS is also triggered by the experimental progress in atom-nanophotonics integration [8-13], as well as the dramatic consequences played by SEBS on the generation of long-range interactions between atoms [14-16].

In this work, we concentrate on a set of boson impurity models described by a Hamiltonian of the form

Published by the American Physical Society under the terms of the Creative Commons Attribution 3.0 License. Further distribution of this work must maintain attribution to the author(s) and the published article's title, journal citation, and DOI.
$H=\Delta \sigma_{e e}+\sum_{\mathbf{k}} \varepsilon_{\mathbf{k}} a_{\mathbf{k}}^{\dagger} a_{\mathbf{k}}+\frac{\Omega}{\sqrt{V}} \sum_{\mathbf{k}} \eta_{\mathbf{k}}\left(a_{\mathbf{k}}^{\dagger} \sigma_{g e}+\right.$ H.c. $)$.

Here, the two-level impurity has ground state $|g\rangle$ and excited state $|e\rangle, \sigma_{e e}=|e\rangle\langle e|$ is the occupation number operator of the excited state, $\sigma_{g e}=|g\rangle\langle e|$ is the transition operator from the $|e\rangle$ state to the $|g\rangle$ state (its Hermitian conjugate is for the opposite process), $V$ is the volume of the bosonic bath, $a_{\mathbf{k}}$ is the annihilation operator of the bath mode with momentum $\mathbf{k}$, and $\eta_{\mathbf{k}}$ is the momentum-dependent coupling profile (typically represented by very smooth functions of $\mathbf{k}$ ) satisfying the normalization condition $\sum_{\mathbf{k}}\left|\eta_{\mathbf{k}}\right|^{2} / V=1$. The most relevant parameters are the detuning $\Delta$, the coupling strength $\Omega \geq 0$, and the dispersion relation $\varepsilon_{\mathbf{k}}$ that we assume to describe a single band of width $W$. In the quantum optical context, $\Delta$ describes the detuning between the two-level transition and the lowest energy of the bath Hamiltonian, and $\Omega$ the coupling strength in the rotatingwave approximation limit. The third term thus describes the process in which the emitter is deexcited by emitting a photon into the bath. The Hamiltonian [Eq. (1)] models a number of relevant problems, ranging from spontaneous emission of a free atom, to the coupling of an emitter to a photonic crystal in any spatial dimension, as well as a single emitter in a high- $Q$ cavity [Jaynes-Cummings (JC) model], which is recovered in the limit of $W \rightarrow 0$.

More than 20 years ago, it was discovered that for certain dispersion relations [1-3] this Hamiltonian displays an exact eigenstate of the form

$$
\left|B_{1}\right\rangle=\alpha|e\rangle|0\rangle+\beta C^{\dagger}|g\rangle|0\rangle,
$$

where $|0\rangle$ is the vaccum state of the bath and $C$ is a collective bath mode given by a linear superposition of the 
$a_{\mathbf{k}}$ 's. In particular, the boson density vanishes far away from the position of the impurity, so that one can interpret that the boson is trapped by the impurity. The length at which this happens, the localization length, depends on the parameters of the model. In recent years, bound states with two excitations have been predicted for some particular dispersion relations [17-22]. Furthermore, there is strong numerical evidence that analogous models [23,24] (where the number of excitations is not conserved) may also possess bound states involving multiple excitations. Thus, some questions that naturally arise are, does Hamiltonian Eq. (1) possess multiple excitation bound states, and if so, what is their origin, how can they be described precisely, and how can they be observed experimentally?

In this paper, we address all of these questions. First of all, we show that for very generic dispersion relations, the boson impurity model Eq. (1) may support infinitely many bound states corresponding to different numbers of excitations. We investigate the origin of those bound states in the limit $|\Delta| \gg W, \Omega$, where one can view the impurity as creating a potential for the bosonic bath, in which the excitations may Bose-Einstein condense. We also postulate a three-parameter family of (approximate) wave functions for the bound states in the form

$$
\left|B_{N}\right\rangle=\left(\alpha|e\rangle\langle g|+\beta C^{\dagger}\right) A^{\dagger N-1}|g\rangle|0\rangle,
$$

where $A$ and $C$ are linear combinations of the $a_{\mathbf{k}}$ 's that depend on the system parameters. We confirm that those wave functions provide a very accurate description of the MEBS for a specific model in one spatial dimension by comparing their physical properties with the results obtained using advanced density-matrix renormalization group (DMRG) techniques $[25,26]$. In this model, we investigate the properties of the MEBS for all parameter regimes, and discover a region that cannot be described perturbatively, in which the energy and correlation length of the bound states are nonanalytical functions of $\Omega$. Additionally, we give exact expressions for the bound states for up to three excitations in the general case. Finally, we propose two different setups where the bound states could be prepared and observed. The first one uses atoms in optical lattices, where the role of the impurity is played by the absence or presence of an atom in an internal state, and the bath by the atoms in another internal state. The dispersion relation can be designed by choosing the geometry of the lattice, and the value of the two other parameters, $\Delta$ and $\Omega$, can be easily tuned by changing the laser frequency and intensity. The second one corresponds to the scenario of an atom coupled to a photonic crystal, and it will be much harder to observe. However, in view of the rapid experimental progress in different fronts [8-13], it is not unforeseeable that some of those states or their consequences could also be observed in the near future.

The paper is structured as follows: In Sec. II, we introduce the general model and requirements that we use throughout the paper. In Sec. III, we derive the conditions for the existence of the MEBSs and find analytical solutions in certain cases, which motivate us to introduce a variational Ansatz for the MEBSs. To make the discussions sufficiently compact, we skip some inessential steps in the main text and the full details are presented in the Appendixes. Then, in Sec. IV, we thoroughly study a particular example of a one-dimensional bath with $\cos (k)$ dispersion relation and find excellent agreement between the variational Ansatz and the DMRG results. We explore the parameter space and reveal the existence of different regimes, yielding different scaling of the energies and localization lengths of the MEBS with the relevant parameters of the system. Finally, in Sec. V, we show how to prepare and detect these MEBSs in two different implementations, and in Sec. VI, we conclude by summarizing the main results of the paper.

\section{MODEL}

The very generic model that we use throughout the paper is schematically depicted in Fig. 1. It consists of three parts: a single impurity, a $d$-dimensional bath of free bosons, and the coupling between impurity and bath. They correspond to the three terms in the Hamiltonian [Eq. (1)]; i.e., $H=H_{\text {imp }}+H_{\text {bath }}+H_{\text {int }} . \quad H_{\text {imp }}=\Delta \sigma_{e e}$ describes the two-level impurity, and the operators $\sigma_{\mu \nu}=|\mu\rangle\langle\nu|$ are used frequently in our discussions. $H_{\text {bath }}=\sum_{\mathbf{k}} \varepsilon_{\mathbf{k}} a_{\mathbf{k}}^{\dagger} a_{\mathbf{k}}$ describes the bath with volume $V$ and energy dispersion $\varepsilon_{\mathbf{k}}$, where $a_{\mathbf{k}}^{\dagger}\left(a_{\mathbf{k}}\right)$ is the creation (annihilation) operator with momentum $\mathbf{k}$. Without loss of generality, we assume that the spectrum has a lower bound $\min \left|\varepsilon_{\mathbf{k}}\right|=0$, but its bandwidth $W$ may be finite or infinite (e.g., in the case of $\varepsilon_{\mathbf{k}} \sim|\mathbf{k}|^{2}$ spectrum). $H_{\text {int }}=\Omega \sum_{\mathbf{k}} \eta_{\mathbf{k}}\left(a_{\mathbf{k}}^{\dagger} \sigma_{g e}+\right.$ H.c. $) / \sqrt{V}$ encodes the coupling between impurity and bath with a strength parametrized by $\Omega$. It can be written in the coordinate space as

$$
H_{\mathrm{int}}=\Omega \sum_{j} \eta_{j}\left(a_{j}^{\dagger} \sigma_{g e}+\text { H.c. }\right),
$$

where $a_{j}=\sum_{\mathbf{k}} a_{\mathbf{k}} e^{i \mathbf{k} \cdot \mathbf{r}_{j}} / \sqrt{V}$ is the annihilation operator of the bosonic bath mode at position $\mathbf{r}_{j}$ and

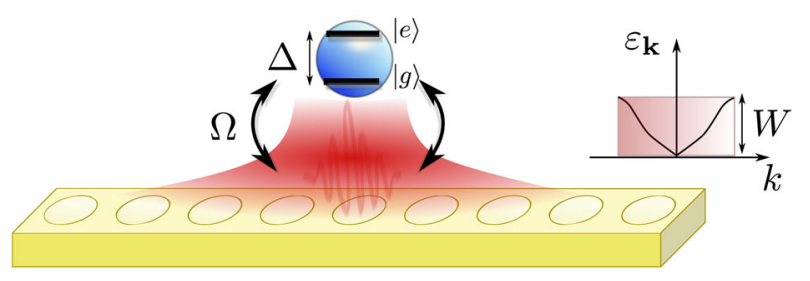

FIG. 1. Single impurity with energy $\Delta$ coupled through $\Omega$ to a bath with dispersion relation $\varepsilon_{\mathbf{k}}$ and a bandwidth $W$. A bosonic bound state (in red) localizes around the impurity. 
$\eta_{j}=\sum_{j} \eta_{\mathbf{k}} e^{i \mathbf{k} \cdot \mathbf{r}_{j}} / V$ is the Fourier transform of the momentum space coupling profile.

It is worth emphasizing that this class of models is ubiquitous and appear in systems ranging from atom coupled to photonic crystal waveguides [1-3,14-16], superconducting qubits coupled to microwave resonators [27], and cold atoms in state-dependent optical lattices $[28,29]$, as we show in Sec. V when we discuss the preparation and detection of the MEBSs. Among the different implementations, a one-dimensional bath with dispersion relation $\varepsilon_{k}=2 J[1-\cos (k)]$ and $\eta_{j}=\delta_{j 0}$ (this can be obtained in a tight-binding model with nearestneighbor hopping) is especially attractive due to recent developments in atom waveguide QED systems [8-13]. Its properties are studied in detail using variational and numerical methods in Sec. IV.

\section{CONDITIONS FOR THE EXISTENCE OF BOUND STATES}

In this section, we derive the conditions for the existence of bound states for arbitrary dispersion relations and spatial dimensions. The Hamiltonian $H$ commutes with the total excitation number operator $N=\sigma_{e e}+\sum_{\mathbf{k}} a_{\mathbf{k}}^{\dagger} a_{\mathbf{k}}$, so we can study the subspaces with different $N$ separately. We first revisit the bound state of one boson $[1,14-16,30,31]$ in Sec. III A in a way more general than what previous works did. For the more interesting cases with multiple bosons, we divide the parameter space into several regimes, as depicted in Fig. 2. The division is based on the methods that we use to understand them as well as the different scaling behaviors of the physical quantities. Using analytical methods, we prove in Sec. III B that bound states exist in certain regimes. These results motivate us to introduce a variational Ansatz in Sec. IIIC that can be used in all regimes.

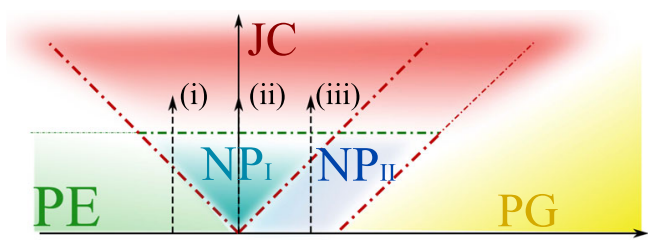

FIG. 2. Parameter space of the boson impurity model as a function of $\Omega$ and $\Delta$. Different colors denote regimes with different EBS origin: perturbative excited (PE, green), perturbative ground (PG, yellow), nonperturbative (NP, blue), and JaynesCummings (JC, red). The boundaries between these regimes are not sharp and depend on the excitation number $N$. See the main text for more details. For the one-dimensional tight-binding model with $\cos (k)$ spectrum, the NP regime is further divided into two subregimes $\mathrm{NP}_{\mathrm{I}}$ and $\mathrm{NP}_{\mathrm{II}}$ (light and dark tones) characterized by different scaling behaviors. The three dashed arrows (i)-(iii) denote the paths that we use in Figs. 3 and 4.

\section{A. Single-excitation bound state}

This case has been considered before in the literature, but mainly focusing on baths with quadratic $\left(\epsilon_{\mathbf{k}} \sim|\mathbf{k}|^{2}\right)$ or tight-binding $\left[\epsilon_{\mathbf{k}} \sim \sum_{i} \cos \left(\mathbf{k}_{i}\right)\right]$ dispersions in different spatial dimensions $[1,14-16,30]$. However, it is instructive to revisit this problem because we can find the conditions for the existence of bound states independent of the model. The wave function of one boson in the system can be written as

$$
\left|B_{1}\right\rangle=u_{e}|e\rangle|0\rangle+\sum_{\mathbf{k}} f_{\mathbf{k}} a_{\mathbf{k}}^{\dagger}|g\rangle|0\rangle
$$

where $|0\rangle$ is the vacuum state of the bath. Bound states would appear if the secular equation $H\left|B_{1}\right\rangle=E_{1}\left|B_{1}\right\rangle$ has solutions that lie outside of the bath spectrum, i.e., $E_{1}<$ $\min \left|\varepsilon_{\mathbf{k}}\right|$ or $E_{1}>\max \left|\varepsilon_{\mathbf{k}}\right|$. For concreteness, we focus on the low-energy SEBS with $E_{1}<\min \left|\varepsilon_{\mathbf{k}}\right|$, but the method we present below can also be used to derive the conditions for the upper ones if the bandwidth $W$ is finite.

By defining the function

$$
F_{1}(E)=E-\Delta-\Omega^{2} \int \frac{d^{d} \mathbf{k}}{(2 \pi)^{d}} \frac{\left|\eta_{\mathbf{k}}\right|^{2}}{E-\varepsilon_{\mathbf{k}}},
$$

the existence condition of SEBS is that $F_{1}(E)=0$ must have solutions that lie outside of the bath spectrum. One can show that $F_{1}(E)$ has two properties: (i) it is a monotonically increasing function and (ii) $F_{1}(E) \rightarrow-\infty$ as $E \rightarrow-\infty$. Thus, if we have $F_{1}(0)>0$, there must be only one solution to $F_{1}(E)=0$, and a unique bound state with $E_{1}<0$ appears. Therefore, the existence or absence of a bound state is essentially determined by the integral

$$
I_{0}=\int \frac{d^{d} \mathbf{k}}{(2 \pi)^{d}} \frac{\left|\eta_{\mathbf{k}}\right|^{2}}{\varepsilon_{\mathbf{k}}} .
$$

For example, for one- and two-dimensional baths with tight-binding and quadratic dispersions, $I_{0} \rightarrow \infty$ due to an infrared divergence (as long as $\left|\eta_{\mathbf{k}}\right|^{2}$ does not vanish at $|\mathbf{k}|=0$ or is not a highly oscillatory function). In these cases, there always exists a SEBS irrespective of $\Delta, \Omega$, or $W$. However, if $I_{0}$ converges to a finite positive value, which happens for a three-dimensional bath with tightbinding dispersion $\varepsilon_{\mathbf{k}}=J\left[6-2 \cos \left(k_{x}\right)-2 \cos \left(k_{y}\right)-\right.$ $\left.2 \cos \left(k_{z}\right)\right]\left(I_{0}=0.253 / J\right.$ in this case $)$, a bound state exists only if $F_{1}(0)=-\Delta+\Omega^{2} I_{0}>0$.

\section{B. Multiple-excitations bound states in strong- and weak-coupling regimes}

The analysis presented above can be extended to systems with multiple excitations. The wave function in the subspace with $N$ excitations can be written as 


$$
\left|B_{N}\right\rangle=\left|\Psi_{e}\right\rangle|e\rangle+\left|\Psi_{g}\right\rangle|g\rangle,
$$

where the two states $\left|\Psi_{e}\right\rangle$ and $\left|\Psi_{g}\right\rangle$ dress the impurity atom when it is in $|e\rangle$ and $|g\rangle$, respectively. The existence of bound states and their properties can be understood analytically in some of the regimes depicted Fig. 2, where the couplings are very strong or very weak.

For example, when the impurity-bath coupling strength $\Omega$ is much larger than the detuning $|\Delta|$ and the bandwidth $W$ (the red regime in Fig. 2), the dominant contribution to the Hamiltonian is the coupling between the impurity and a collective bath mode $\sum_{j} \eta_{j} a_{j}^{\dagger}$, so the physics is the same as that of the well-known JC model [32].

For the opposite cases, where the impurity-bath coupling is weak, such that $\Omega \ll|\Delta|$ and $\Omega \ll \Delta-W$ (the darker green and yellow regimes in Fig. 2), the coupling $H_{\text {int }}$ is a small perturbation to both the impurity and bath so we can eliminate it to the first order of the coupling strength $\Omega$ using a Fröhlich transformation, which leads to the effective Hamiltonian $H_{\text {eff }}=\tilde{\Delta} \sigma_{e e}+H_{\text {bath }}+V_{\text {eff }}$. It shows a very clear physical picture: (i) the impurity energy $\Delta$ acquires a Lamb shift and changes to

$$
\tilde{\Delta}=\Delta+\frac{\Omega^{2}}{V} \sum_{\mathbf{k}} \frac{\left|\eta_{\mathbf{k}}\right|^{2}}{\Delta-\varepsilon_{\mathbf{k}}},
$$

and, more importantly, (ii) a nonlocal potential

$$
V_{\text {eff }}=\frac{\Omega^{2}}{2 V} \sum_{\mathbf{k}, \mathbf{k}^{\prime}}\left(\frac{\eta_{\mathbf{k}^{\prime}}^{*} \eta_{\mathbf{k}}}{\Delta-\varepsilon_{\mathbf{k}^{\prime}}}+\frac{\eta_{\mathbf{k}^{\prime}}^{*} \eta_{\mathbf{k}}}{\Delta-\varepsilon_{\mathbf{k}}}\right) a_{\mathbf{k}}^{\dagger} a_{\mathbf{k}^{\prime}} \sigma_{z}
$$

is generated $\left[\sigma_{z}=\left(\sigma_{e e}-\sigma_{g g}\right)\right]$, which localizes the excitations around the impurity to form a MEBS.

We term the perturbative regime with negative (positive) detuning $\Delta$ [see the green (yellow) regime in Fig. 2] as excited (ground) because in this regime the $\left|\Psi_{e}\right\rangle|e\rangle$ $\left(\left|\Psi_{g}\right\rangle|g\rangle\right)$ part is the dominant one in the ground-state wave function Eq. (8). For this reason, the effective Hamiltonian can be projected to the $|e\rangle$ and $|g\rangle$ state in the perturbative excited (PE) and perturbative ground (PG) regimes, respectively, which gives the Hamiltonians $H_{\text {eff }}^{e}$ and $H_{\text {eff }}^{g}$ for the two regimes. The bath components $\left|\Psi_{e}\right\rangle \sim A_{e}^{\dagger N-1}|0\rangle$ in the PE regime and $\left|\Psi_{g}\right\rangle \sim A_{g}^{\dagger N}|0\rangle$ in the PG regime, where $A_{e}^{\dagger}$ and $A_{g}^{\dagger}$ are collective bosonic bath operators determined by the secular equation $H_{\text {eff }}^{s} A_{s}^{\dagger}|0\rangle=E_{1}^{s} A_{s}^{\dagger}|0\rangle$ ( $s=e$ or $g$ ). Although we focus on MEBS, which are ground states of their respective $N$-excitation manifolds, there may also exist excited states that are bound to the impurity (e.g., in the PG regime) with the state $|e\rangle$ being dressed by a localized bosonic mode.

The bosons form a bound state if the secular equation have solutions with energy values $E_{1}^{s}<0$. As for the case with one excitation, the existence condition can also be formulated using two functions $F_{N}^{s}(E)$ that depend on the integral Eq. (7). If $I_{0} \rightarrow+\infty$ due to infrared divergence, we have MEBS in both regimes. For the PE regime, the ground state

$$
\left|B_{N}\right\rangle=\frac{A_{e}^{\dagger N-1}|0\rangle|e\rangle}{\sqrt{(N-1) !}}+\frac{C_{e}^{\dagger} A_{e}^{\dagger N-1}|0\rangle|g\rangle}{\sqrt{(N-1) !}}
$$

has energy $E_{N}^{e}=E_{1}+(N-1) E_{1}^{e}$. For the PG regime, the ground state

$$
\left|B_{N}\right\rangle=\frac{d_{g} A_{g}^{\dagger N-1}|0\rangle|e\rangle}{\sqrt{(N-1) !}}-\frac{A_{g}^{\dagger N}|0\rangle|g\rangle}{\sqrt{N !}}
$$

has energy $E_{N}^{g}=N E_{1}^{g}$. The full details of the perturbative analysis are given in Appendix A.

The analysis presented above relies on perturbation theory, but the bound states survive even if we move to the nonperturbative (NP) regimes (light and dark blue regimes in Fig. 2), where the detuning $\Delta$, the coupling strength $\Omega$, and the bandwidth $W$ do not satisfy the perturbative conditions used above. In fact, increasing $\Omega$ to be larger than $|\Delta|$ for all values of $\Delta$ or immersing $\Delta$ within the band (i.e., $0<\Delta<W$ ) make the bound states more localized. This is confirmed in Appendix B using a quantum field theoretical approach in the subspaces with excitation number $N=2,3$. In summary, we conclude that the MEBS exist in all parameter regimes if $I_{0} \rightarrow+\infty$.

For baths with tight-binding and quadratic dispersions, $I_{0}$ diverges in one and two dimensions but converges in three dimensions. This means that SEBS and MEBS exist in all regimes in one and two dimensions, but they only emerge if the coupling strength $\Omega$ is larger than a critical value (which depends on the detuning $\Delta$ ) in three dimensions. Moreover, because $F_{N}^{s}(E)$ and $F_{1}(E)$ have very different forms, we may have only SEBS but not MEBS in certain regimes in three dimensions.

\section{Variational Ansatz for multiple-excitation bound states in all regimes}

In the previous section, we show that the existence of the MEBS can be guaranteed analytically in certain regimes with very strong or very weak couplings $\Omega$ for all excitation numbers $N$. However, it is obviously desirable to have a way of describing the whole parameter space of Fig. 2, including those denoted as nonperturbative regimes (in light and dark blue) where both the strong- and weakcoupling expansions fail.

Inspired by the perturbative solutions of Eqs. (11) and (12) as well as the exact solutions for $N=2$ and 3, we introduce the following variational Ansätze for the ground and excited bath components of $\left|B_{N}\right\rangle$ : 


$$
\begin{aligned}
& \left|\Psi_{e}\right\rangle=\alpha \frac{A^{\dagger N-1}}{\sqrt{(N-1) !}}|0\rangle, \\
& \left|\Psi_{g}\right\rangle=\left[\beta \frac{A^{\dagger N}}{\sqrt{N !}}+\gamma \frac{A^{\dagger N-1} B^{\dagger}}{\sqrt{(N-1) !}}\right]|0\rangle,
\end{aligned}
$$

where $\alpha, \beta, \gamma$ are variational parameters that allow us to interpolate between the different parameter regimes and $A^{\dagger}$, $B^{\dagger}=\sum_{\mathbf{k}} \varphi_{A, B}(\mathbf{k}) a_{\mathbf{k}}^{\dagger}$ are two orthonormal collective modes of the bath.

By minimizing the energy $E_{\text {g.s. }}=\left\langle B_{N}|H| B_{N}\right\rangle$ of the variational Ansätze under the normalization condition $|\alpha|^{2}+|\beta|^{2}+|\gamma|^{2}=1$ and the orthonormal constraints $\left[A, A^{\dagger}\right]=\left[B, B^{\dagger}\right]=1$ and $\left[A, B^{\dagger}\right]=0$, we can determine the parameters $\alpha, \beta, \gamma$ and the mode functions $\varphi_{A, B}(\mathbf{k})$. These mode functions are superpositions of $\eta_{\mathbf{k}} /\left(e_{1,2}-\varepsilon_{\mathbf{k}}\right)$ ( $e_{1,2}$ are two negative variables), and their Fourier transform in coordinate space can reveal the localization properties of the MEBS. The full details of this process are given in Appendix C.

\section{ONE-DIMENSIONAL TIGHT-BINDING BATH: VARIATIONAL AND EXACT RESULTS}

In this section, we study a particularly relevant model for which the bath is a one-dimensional lattice described by the tight-binding Hamiltonian $H_{\text {bath }}=-J \sum_{\langle j m\rangle}\left(a_{j}^{\dagger} a_{m}+\right.$ H.c. $)+2 J \sum_{j} a_{j}^{\dagger} a_{j}$, where $\langle j m\rangle$ denotes nearest neighbors. The central site of the bath is labeled as the zeroth one and those on its left (right) are labeled by negative (positive) integers. The coupling between impurity and bath occurs only on the zeroth site, i.e., $\eta_{j}=\delta_{j 0}$. We compute the ground states of this model using the DMRG algorithm $[25,26]$ in the $N=2,3,4,5$ subspaces. We use open boundary conditions as they are more suitable for the algorithm, but the boundary effect is negligible when the number of lattice sites is large enough (we have studied systems with up to 1000 bath sites).

To confirm the validity of our variational Ansätze, we compare some physical quantities given by variational and DMRG approaches. In addition to the ground-state energy, we also study the experimentally measurable localization lengths of the bath components defined as ( $s=e$ or $g$ )

$$
\xi_{s}=\sqrt{\frac{\sum_{j} j^{2}\left\langle\Psi_{s}\left|a_{j}^{\dagger} a_{j}\right| \Psi_{s}\right\rangle}{\sum_{j}\left\langle\Psi_{s}\left|a_{j}^{\dagger} a_{j}\right| \Psi_{s}\right\rangle} .}
$$

We choose three different paths in the parameter space labeled as (i)-(iii) in Fig. 2, which cover a wide range of coupling strengths $\Omega$ at three different detunings $\Delta$. In Fig. 3, we compare the modified ground-state energies $\tilde{E}_{N}=\left|E_{N}-\Theta(-\Delta) \Delta\right|$ [where $\Theta(x)$ is the Heaviside step function] given by variational (solid lines) and DMRG (markers) calculations for the $N=3,4$ cases. In Fig. 4, we

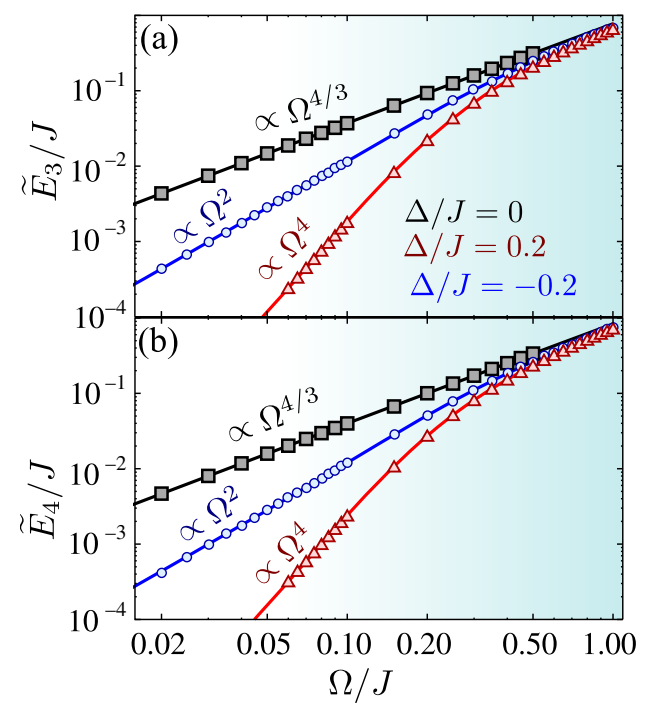

FIG. 3. Comparison of the bound state energies $\tilde{E}_{N}$ given by the variational Ansätze (solid lines) with infinite bath size and the DMRG results (markers) in the (a) $N=3$ and (b) $N=4$ subspaces. The three different curves correspond to the three different paths depicted in Fig. 2, i.e., choosing a detuning $\Delta / J=-0.2, \Delta / J=0$, and $\Delta / J=0.2$.

compare the localization lengths given by variational (solid lines) and DMRG (markers) calculations for the $N=2,3$, 4, 5 cases. The perfect agreement in all cases clearly demonstrates the power of our variational Ansatz.

We can provide a more detailed characterization of Fig. 2 by establishing scaling relations of physical quantities. This can be done using variational Ansatz and DMRG results, which leads to the scaling relations presented in Table I.

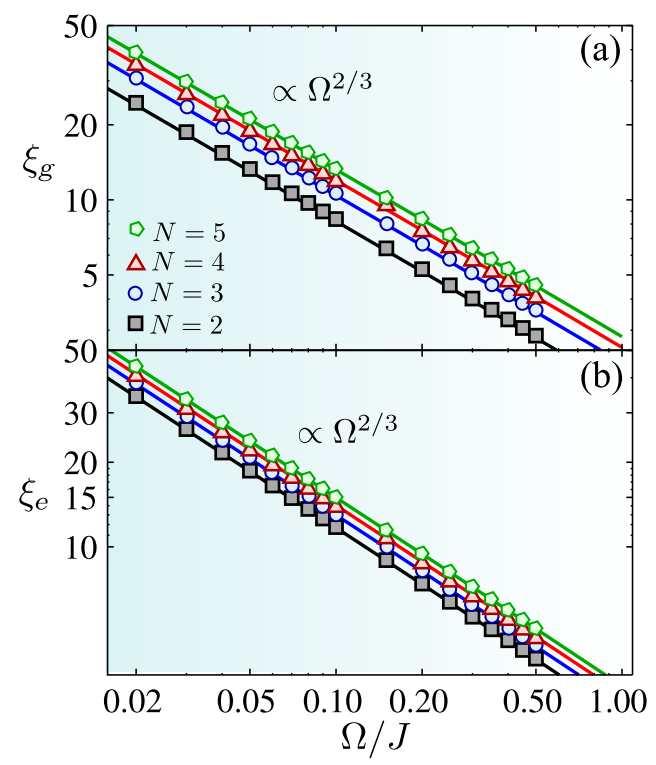

FIG. 4. Comparison of the localization lengths (a) $\xi_{g}$ and (b) $\xi_{e}$ given by the variational Ansätze (solid lines) with infinite bath size and the DMRG results (markers) with 1000 bath sites at $\Delta / J=0$ in the $N=2,3,4,5$ subspaces. 
TABLE I. Scaling analysis of the relevant properties of EBS for the regimes with $\Omega \ll W$ depicted in Fig. 2 .

\begin{tabular}{cccc}
\hline \hline & $\mathrm{PE}$ & $\mathrm{NP}_{\mathrm{I}}$ & $\mathrm{NP}_{\mathrm{II}}, \mathrm{PG}$ \\
\hline$E_{N}$ & $\Delta-\left[\left(\Omega^{2}\right) / \sqrt{|\Delta|(|\Delta|+4 J)}\right]$ & $\propto \Omega^{4 / 3}$ & $\propto N \Omega^{4}$ \\
$|\alpha|$ & $1-d_{\gamma}^{2} \Omega^{2}$ & $\mathrm{O}(1)$ & $d_{\alpha} \Omega^{2}$ \\
$|\beta|$ & $\propto \Omega^{2}$ & $\mathrm{O}(1)$ & $1-d_{\alpha} \Omega^{4}$ \\
$|\gamma|$ & $d_{\gamma} \Omega$ & $\mathrm{O}(1)$ & $\propto \Omega^{4}$ \\
\hline \hline
\end{tabular}

However, we should be careful when trying to extract the scaling exponents, as they are well defined only in certain limits. Therefore, Fig. 2 should not be understood as a phase diagram but rather as an indication of the different scaling behaviors of the system, which are only well defined in the corresponding limits. This means that the boundaries between different regimes are not sharply defined (unlike the boundary in a quantum phase transition).

To illustrate the different behaviors, we focus the discussion on the three paths depicted in Fig. 2, as these represent the experimentally most relevant regimes that occur when $\Omega \ll W$. For path (i) with $\Delta / J=-0.2$, the system changes from the PE regime with $\tilde{E}_{N} \propto \Omega^{2}$ to the $\mathrm{NP}_{\mathrm{I}}$ regime. This change is also manifested in the variational parameters: we have $1-|\alpha| \propto \Omega^{2}$ in the PE regime (so most of the weight in the wavefunction comes from $\left|\Psi_{e}\right\rangle$ ) but $|\alpha|$, $|\beta|,|\gamma|$ of the same order $\sim O(1)$ in the $\mathrm{NP}_{\mathrm{I}}$ regime. For path (ii) with $\Delta / J=0$, we move only within the $\mathrm{NP}_{\mathrm{I}}$ regime. For path (iii) with $\Delta / J=0.2$, the system changes from the $\mathrm{NP}_{\mathrm{II}}$ regime with $\tilde{E}_{N} \propto N \Omega^{4}$ to the $\mathrm{NP}_{\mathrm{I}}$ regime. $\tilde{E}_{N}$ in the $\mathrm{NP}_{\mathrm{I}}$ regime exhibits nonanalytical behavior because it scales as a fractional power of $\Omega$ in this regime. The exponent is only well defined if $\Omega \ll|\Delta|$, which is satisfied by path (ii) where $\tilde{E}_{N} \propto \Omega^{4 / 3}$, but not by paths (i) and (iii). The MEBS in the $\mathrm{NP}_{\mathrm{II}}$ regime can be understood as a Gutzwiller projected condensate. To see this, we note that $1-|\beta| \propto \Omega^{4}$, so the state basically represents $N$ bosons in the $A$ mode. It can be constructed by first putting all the bosons in the SEBS $\left|B_{1}\right\rangle$ and then projecting out the configurations with more than one boson in the $|e\rangle$ state. Because the scaling relations may depend on the excitation number $N$, the crossovers between different regimes in the parameter space could also be $N$ dependent.

To conclude this section, we show that a closer inspection of the DMRG results provides further insight into the structure of the MEBS. We interpret the impurity as a lattice site described by hard-core boson operator $a_{\mathrm{hc}}$ and compute the two-point correlation functions

$$
G_{j m}=\left\langle B_{N}\left|a_{j}^{\dagger} a_{m}\right| B_{N}\right\rangle,
$$

where $j, m$ run over all the bath sites as well as the impurity site. It is found that the eigenvalues of the matrix $G_{j m}$ have only two dominant eigenvalues $p_{ \pm}$in all parameter regimes and for all values of $N$ (see Fig. 5 for some examples). This

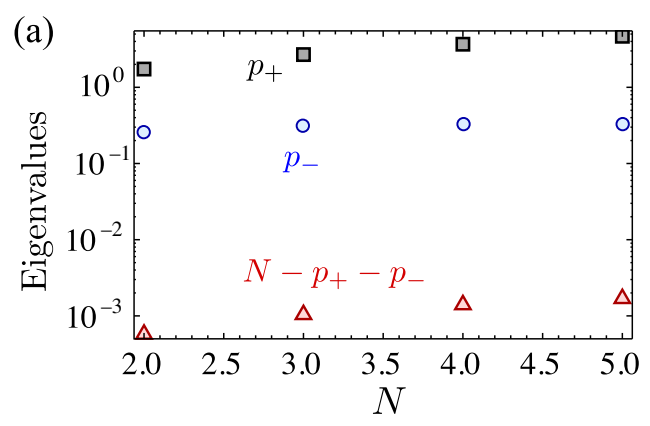

(b)

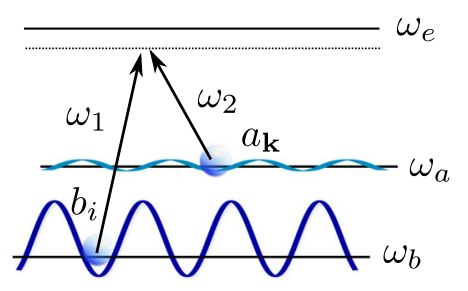

FIG. 5. (a) Eigenvalues of the correlation matrix are shown for multi-EBS as a function of $N$ for $\Delta / J=0$ and $\Omega / J=0.5$. (b) Scheme of the implementation with state-dependent optical lattices: two atomic states $a_{i}, b_{j}$ are trapped in a shallow or deep potential and connected through a two-photon Raman transition.

implies that the MEBS $\left|B_{N}\right\rangle$ mainly lives in the symmetric space defined by two orthonormal modes. Without loss of generality, these two modes can be chosen as $A_{1}=A$ and $A_{2}=a_{\mathrm{hc}} \cos \theta+B \sin \theta$, and the bound state can then be approximated as

$$
\left|B_{N}\right\rangle=\sum_{n=0}^{N} \alpha_{n} \frac{\left(A_{1}^{\dagger}\right)^{N-n}\left(A_{2}^{\dagger}\right)^{n}}{\sqrt{(N-n) ! n !}}|0\rangle .
$$

The hard-core nature of the impurity imposes the constraint that $\alpha_{n}=0$ for $n>1$, which recovers the variational Ansätze given in Eq. (13). Indeed, the two eigenvalues computed from Eq. (13) are $p_{ \pm}=\left[N \pm \sqrt{N^{2}-4(N-1)\left(1-\beta^{2}\right)^{2}}\right] / 2$.

\section{PREPARATION AND DETECTION OF MULTIPARTICLE BOUND STATES}

We consider a very general model of a single impurity coupled to a bosonic bath that may be implemented in a plethora of different systems, ranging from superconducting circuits [27], atoms near photonic crystals [1,8$13,15,16]$, or cold atoms in optical lattices [28,29]. In this section, we show how to prepare and detect them in two of these platforms.

\section{A. Optical emitters coupled to photonic reservoirs}

The observation of bound states in optical platforms is challenging because typically the coupling strength is much smaller than the associated bandwidth $(\Omega \ll W)$ and losses of the photons $(\kappa)$ or the excited state of the impurity $(\Gamma)$, 
which give rise to a finite lifetime of the MEBS. One option is to use multiphoton scattering states, which was explored with a single photon in Refs. [18,19,21-23,31]. Another way of circumventing these limitations is using atomic detection and postselection at the expense of making the process probabilistic. The procedure to prepare a given $\left|B_{N}\right\rangle$ is to apply a sequence of $\pi$ pulses on the impurity and perform postselection by projecting in the atomic state $P_{s}=|s\rangle\langle s|$, with $s=g$, e. We start with the impurity in the ground state and no photons, i.e., $\left|\Psi_{0}\right\rangle=|g\rangle \otimes\left|0_{k}\right\rangle$, and focus on a situation with $\Delta<0$ and $|\Delta| \gg \Omega$. Then, if we apply a $\pi$ pulse on the impurity and let it evolve, the atom will mainly follow $\left|\Psi_{0}\right\rangle \rightarrow|e\rangle \otimes\left|0_{k}\right\rangle \rightarrow\left|B_{1}\right\rangle$, where the contribution of scattering states will be very small because we are in a region where there are no modes to decay into. Then, we measure the impurity state $P_{g}$, and apply another $\pi$ pulse if we detect an excitation; this will induce the change to $P_{g}\left|B_{1}\right\rangle \rightarrow|e\rangle \otimes\left|1_{k}\right\rangle$. As the state $|e\rangle \otimes\left|1_{k}\right\rangle$ is within the two-excitation subspace, it evolves to $\left|B_{2}\right\rangle$. To continue building up $\left|B_{N}\right\rangle$, we need to apply the sequence of $P_{g}$ measurements and $\pi$ pulse $N-1$ times to arrive at the desired excitation number $N$. Obviously, these sequences of $\pi$ pulses and postselections must be faster than the lifetime determined by $\Gamma, \kappa$ such that the MEBS survives at the end of the process. For this reason, circuit QED [27], though in the microwave regime, can be a better platform to observe the MEBS because both photon and qubit lifetimes are longer than those at optical setups.

\section{B. Cold atoms in state-dependent optical lattices}

Because of the limitations of standard quantum optical setups, one can think of using cold atoms trapped in optical lattices to simulate this kind of Hamiltonian, as originally proposed in Refs. [28,29]. This can be done by using statedependent optical lattices in which two atomic states experience very different trapping potentials that can be obtained, e.g., using alkaline-earth atoms like ytterbium $[33,34]$. These lattices can be designed in a way such that the two atomic states experience a very shallow or deep potential, respectively. The ones in the shallow potential $\left(a_{j}\right)$ have large tunneling amplitude $J$ and play the role of the bosonic bath; on the contrary, the ones in the deep potential will be localized and serve as the impurity $\left(b_{j}\right)$. Moreover, we can tune the onsite interaction $U$ of the impurity states to $\infty$ such that it behaves effectively as a two-level system. The coupling between the two may be achieved through an off-resonant Raman transition to a common excited state yielding an effective number-conserving Hamiltonian. Interestingly, the coupling $\Omega$ and detuning $\Delta$ can be controlled independently of $J$ by the Raman parameters, which allows us to explore the whole parameter space in Fig. 2. Moreover, using the recently developed single-atom resolution microscopy and addressing techniques, we can achieve the single impurity regime and thus we can simulate the Hamiltonian $H[28,29]$. One way of preparing the $\left|B_{N}\right\rangle$ is to start from a situation where the tunneling $J$ is switched off by increasing potential depth of the bath atoms and setting $\Delta$ to zero. Then we load $N$ atoms in the bath mode $a_{0}$ (the one that couples to the impurity) to give an initial state $\left|\Psi_{0}\right\rangle=\left(a_{0}^{\dagger N} / \sqrt{N !}\right)|0\rangle|g\rangle=|N\rangle|g\rangle$. The protocol to prepare $\left|B_{N}\right\rangle$ consists of switching a strong Raman field with a $\pi / 2$ phase, i.e., $\Omega=i|\Omega|$, for a time $t_{N}=$ $3 \pi /(4 \sqrt{N}|\Omega|)$ to reach the state $\left|\Psi\left(t_{N}\right)\right\rangle=(|N-1\rangle|e\rangle-$ $|N\rangle|g\rangle) / \sqrt{2}$. After that, we suddenly change the phase of $\Omega$ to be real such that $\left|\Psi\left(t_{N}\right)\right\rangle$, which coincides with $\left|B_{N}\right\rangle$ in the limit of $\Omega \gg|\Delta|, J$. Once we have such a state, we can change the state adiabatically with $\Delta$ or $J$ to explore the whole parameter space.

\section{CONCLUSIONS}

In summary, we unravel the existence of multiple excitation bound states in a very fundamental model of a single impurity coupled to a bosonic bath through a number-conserving interaction. We first show that in certain regimes the impurity-bath coupling gives rise to an effective potential that is able to localize the particles around the impurity. Moreover, we provide the theoretical tools to characterize the bound states in all the parameter regimes by introducing a variational wave function that works for all energy dispersions and spatial dimensions. We test our variational Ansatz for the case of a onedimensional tight-binding model with exact and numerical calculations up to $N=5$ excitations and are able to distinguish different regimes depending on the scaling behavior of the energies or localization lengths, including one with nonanalytical relations. Finally, we present two state-of-the-art implementations where the preparation and detection of these bound states is promising.

\section{ACKNOWLEDGMENTS}

The work was funded by the European Union Integrated project Simulators and Interfaces with Quantum Systems (SIQS). A. G.-T. also acknowledges support from the Alexander von Humboldt Foundation and Intra-European Marie-Curie Fellowship NanoQuIS (625955).

Note added.-During the submission of the manuscript, we became aware of a related work by Calajo et al. [35] that discusses the emergence of multiphoton bound states in waveguide QED setups.

\section{APPENDIX A: CONDITIONS FOR THE EXISTENCE OF MULTIPARTICLE BOUND STATES}

This appendix provides full details about how to derive the conditions for the existence of MEBS.

In the strong-coupling regime with coupling strength much larger than the other energy scales $(\Omega \gg|\Delta|, W)$, the model is essentially the same as the Jaynes-Cummings model. The ground state 


$$
\left|B_{N}\right\rangle=\frac{1}{\sqrt{\mathcal{N}_{\mathrm{JC}}}}\left(\left|E_{N}\right| A_{\mathrm{JC}}^{\dagger N-1}|e\rangle-\sqrt{N} \Omega A_{\mathrm{JC}}^{\dagger N}|g\rangle\right),
$$

where $E_{N}=\left(\Delta-\sqrt{\Delta^{2}+4 N \Omega^{2}}\right) / 2$ is its energy, $A_{\mathrm{JC}}=$ $\sum_{j} \eta_{j} a_{j}$ is a collective bath mode, and $\mathcal{N}_{\mathrm{JC}}$ is the normalization constant. The other couplings to the bath are weak perturbations that enter to $E_{N}$ as a frequency shift of smaller order than the leading term.

In the perturbative regimes, the effective Hamiltonian Eq. (10) can be obtained by applying the Fröhlich transformation with generating function

$$
S=\frac{\Omega}{\sqrt{V}} \sum_{\mathbf{k}}\left(\frac{\eta_{\mathbf{k}} a_{\mathbf{k}}^{\dagger} \sigma_{g e}}{\Delta-\varepsilon_{\mathbf{k}}}-\frac{\eta_{\mathbf{k}}^{*} a_{\mathbf{k}} \sigma_{e g}}{\Delta-\varepsilon_{\mathbf{k}}}\right)
$$

on the original Hamiltonian. The transformed Hamiltonian $H_{\text {eff }}=e^{-S} H e^{S}$ is computed to the first order of $\Omega$ and results in Eq. (10). The effective Hamiltonian can be projected to the $|e\rangle(|g\rangle)$ state in the $\mathrm{PE}$ (PG) regime to give

$$
\begin{aligned}
H_{\text {eff }}^{s}= & \tilde{\Delta} \sigma_{e e}+H_{\text {bath }} \\
& -\frac{\Omega^{2}}{2 V} \sum_{\mathbf{k}^{\prime}} \eta_{\mathbf{k}^{\prime}}^{*} \eta_{\mathbf{k}}\left(\frac{1}{\left|\Delta-\varepsilon_{\mathbf{k}^{\prime}}\right|}+\frac{1}{\left|\Delta-\varepsilon_{\mathbf{k}}\right|}\right) a_{\mathbf{k}}^{\dagger} a_{\mathbf{k}^{\prime}}
\end{aligned}
$$

$(s=e$ or $g)$.

The projected effective Hamiltonian defines a secular equation $H_{\text {eff }}^{s} A_{s}^{\dagger}|0\rangle=E_{1}^{s} A_{s}^{\dagger}|0\rangle$. The operator $A_{s}$ is a collective bath operator that can be expanded as $\sum_{\mathbf{k}} \varphi_{A}^{s}(\mathbf{k}) a_{\mathbf{k}}$, where the mode functions

$$
\varphi_{A}^{s}(\mathbf{k})=\frac{\Omega^{2} \eta_{\mathbf{k}}}{2\left(E_{1}^{s}-\varepsilon_{\mathbf{k}}\right)}\left(C_{2}-\frac{C_{1}}{\left|\Delta-\varepsilon_{\mathbf{k}}\right|}\right)
$$

and the variables $C_{1}^{s}$ and $C_{2}^{s}$ can be obtained by solving the self-consistent equation $\mathbf{M}^{s}\left(E_{1}^{s}, \Delta\right) \mathbf{C}^{s}=0 . \mathbf{M}^{s}$ is a $2 \times 2$ matrix with elements

$$
\begin{aligned}
& \mathbf{M}_{11}^{s}(E, \Delta)=\mathbf{M}_{22}^{s}(E, \Delta)=1+\frac{w_{1}(E)-w_{1}(\Delta)}{|\Delta-E|}, \\
& \mathbf{M}_{12}^{s}(E, \Delta)=-w_{1}(E), \\
& \mathbf{M}_{21}^{s}(E, \Delta)=-\frac{w_{1}(E)-w_{1}(\Delta)}{(\Delta-E)^{2}}-\frac{\partial_{\Delta} w_{1}(\Delta)}{\Delta-E},
\end{aligned}
$$

where

$$
w_{\alpha}(E)=\frac{\Omega^{2}}{2} \int \frac{d^{d} \mathbf{k}}{(2 \pi)^{d}} \frac{\varepsilon_{\mathbf{k}}^{\alpha-1}\left|\eta_{\mathbf{k}}\right|^{2}}{\left(E-\varepsilon_{\mathbf{k}}\right)^{\alpha}}
$$

and $\mathbf{C}=\left(C_{1}, C_{2}\right)^{T}$.

The MEBS exist if the equation $F_{N}^{s}(E)=\operatorname{det} \mathbf{M}^{\mathbf{s}}\left(E^{s}\right)=$ 0 has solutions $E_{1}^{s}<0$, i.e., they lie outside of the bath spectrum. Because $F_{N}^{s}(E)$ is a continuous function and is negative when $E \rightarrow \infty$, there will be at least one solution if $F_{N}^{s}(0)<0$. From the explicit expression

$F_{N}^{s}(0)=\left[1-\frac{w_{1}(\Delta)}{|\Delta|}\right]^{2}-\left[2+\frac{w_{2}(\Delta)}{|\Delta|}\right] \frac{\Omega^{2} I_{0}}{2|\Delta|}<0$,

we can see that the existence or absence of MEBS depends on $I_{0}$. If $I_{0} \rightarrow+\infty$ due to infrared divergence, we have $F_{N}^{s}(0) \rightarrow-\infty$ and MEBS exist in both regimes.

The bath component of the MEBS in the PE regime is

$$
\left|\Psi_{e}\right\rangle=\frac{A_{e}^{\dagger N-1}|0\rangle}{\sqrt{(N-1) !}},
$$

and the full ground state is

$\left|B_{N}\right\rangle=e^{S}\left|\Psi_{e}\right\rangle|e\rangle=\frac{A_{e}^{\dagger N-1}|0\rangle|e\rangle}{\sqrt{(N-1) !}}+\frac{C_{e}^{\dagger} A_{e}^{\dagger N-1}|0\rangle|g\rangle}{\sqrt{(N-1) !}}$.

The bath component of the MEBS in the PG regime is

$$
\left|\Psi_{g}\right\rangle=\frac{A_{g}^{\dagger N}|0\rangle}{\sqrt{N !}}
$$

and the full ground state is

$\left|B_{N}\right\rangle=e^{S}\left|\Psi_{g}\right\rangle|g\rangle=\frac{d_{g} A_{g}^{\dagger N-1}|0\rangle|e\rangle}{\sqrt{(N-1) !}}-\frac{A_{g}^{\dagger N}|0\rangle|g\rangle}{\sqrt{N !}}$.

The operator $C_{e}^{\dagger}$ and the number $d_{g}$ used above are defined as

$$
\begin{gathered}
C_{e}^{\dagger}=\frac{\Omega}{\sqrt{V}} \sum_{\mathbf{k}} \frac{\eta_{\mathbf{k}} a_{\mathbf{k}}^{\dagger}}{\Delta-\varepsilon_{\mathbf{k}}}, \\
d_{g}=\frac{\Omega \sqrt{N}}{\sqrt{V}} \sum_{\mathbf{k}} \frac{\eta_{\mathbf{k}}^{*} \varphi_{A}^{g}(\mathbf{k})}{\Delta-\varepsilon_{\mathbf{k}}} .
\end{gathered}
$$

The ground-state energy of the MEBS is $E_{N}^{e}=\tilde{\Delta}+(N-1) E_{1}^{e}$ in the PE regime and $E_{N}^{g}=N E_{1}^{g}$ in the PG regime.

\section{APPENDIX B: EXACT RESULTS FOR $N=2$ AND $N=3$}

This appendix provides full details about how to obtain the exact results for the MEBS in the $N=2$ and 3 subspaces using quantum field theory.

For convenience, we introduce a hard-core boson described by the annihilation (creation) operator $a_{\mathrm{hc}} \equiv$ $b_{0}\left(a_{\mathrm{hc}}^{\dagger} \equiv b_{0}^{\dagger}\right)$ and rewrite the Hamiltonian as $H=H_{0}+$ $H_{\mathrm{hc}}$, where $H_{0}$ is a quadratic term 


$$
H_{0}=H_{\text {bath }}+\Delta b_{0}^{\dagger} b_{0}+\frac{\Omega}{\sqrt{V}} \sum_{\mathbf{k}} \eta_{\mathbf{k}}\left(a_{\mathbf{k}}^{\dagger} b_{0}+b_{0}^{\dagger} a_{\mathbf{k}}\right)
$$

and $H_{\mathrm{hc}}$ represents a hard-core interaction

$$
H_{\mathrm{hc}}=\frac{U_{0}}{2} b_{0}^{\dagger 2} b_{0}^{2},
$$

with an infinite strength $U_{0}$. The total particle number $N=$ $b_{0}^{\dagger} b_{0}+\sum_{\mathbf{k}} a_{\mathbf{k}}^{\dagger} a_{\mathbf{k}}$ is still a conserved quantity, so we can diagonalize the Hamiltonian separately in the subspaces with different $N$. For example, the eigenstate for $N=1$ has the form

$$
\left|1_{\lambda}\right\rangle=\left[u_{\lambda} b_{0}^{\dagger}+\sum_{\mathbf{k}} f_{\lambda}(\mathbf{k}) a_{\mathbf{k}}^{\dagger}\right]|0\rangle .
$$

The secular equation is a set of coupled equations

$$
\begin{aligned}
\Delta u_{\lambda}+\frac{\Omega}{\sqrt{V}} \sum_{\mathbf{k}} \eta_{\mathbf{k}} f_{\lambda}(\mathbf{k}) & =E_{1 \lambda} u_{\lambda}, \\
\varepsilon_{\mathbf{k}} f_{\lambda}(\mathbf{k})+\frac{\Omega}{\sqrt{V}} \eta_{\mathbf{k}} u_{\lambda} & =E_{1 \lambda} f_{\lambda}(\mathbf{k}),
\end{aligned}
$$

where $E_{1 \lambda}$ is the energy eigenvalue.

For the bound state energy $E_{1} \notin \varepsilon_{\mathbf{k}}$ outside the continuum $\varepsilon_{\mathbf{k}}$, solving Eq. (B4) gives the wave function

$$
f_{B}(\mathbf{k})=\frac{\Omega}{\sqrt{V}} \frac{\eta_{\mathbf{k}} u_{B}}{E_{1}-\varepsilon_{\mathbf{k}}},
$$

where the normalization factor is

$$
u_{B}^{-2}=1+\int \frac{d^{d} \mathbf{k}}{(2 \pi)^{d}} \frac{\Omega^{2} \eta_{\mathbf{k}}^{2}}{\left(E_{1}-\varepsilon_{k}\right)^{2}},
$$

and the bound state energy satisfies the equation

$$
E_{1}=\Delta+\int \frac{d^{d} \mathbf{k}}{(2 \pi)^{d}} \frac{\Omega^{2} \eta_{\mathbf{k}}^{2}}{E_{1}-\varepsilon_{\mathbf{k}}} .
$$

For a general dispersion relation $\varepsilon_{\mathbf{k}}$, the spectrum $E_{1 \lambda}$ is obtained by numerical diagonalization of

$$
\mathcal{H}_{0}=\left(\begin{array}{cccc}
\Delta & \frac{\Omega}{\sqrt{V}} \eta_{\mathbf{k}_{1}} & \cdots & \frac{\Omega}{\sqrt{V}} \eta_{\mathbf{k}_{N}} \\
\frac{\Omega}{\sqrt{V}} \eta_{\mathbf{k}_{1}} & \varepsilon_{\mathbf{k}_{1}} & 0 & 0 \\
\cdots & 0 & \cdots & 0 \\
\frac{\Omega}{\sqrt{V}} \eta_{\mathbf{k}_{N}} & 0 & 0 & \varepsilon_{\mathbf{k}_{N}}
\end{array}\right),
$$

and its eigenvectors determine the parameters $u_{\lambda}$ and the wave function $f_{\lambda}(\mathbf{k})$.

To analyze the MEBS, it is convenient to introduce the Green functions

$$
\begin{aligned}
G_{b b}^{(0)}(t) & \equiv G_{b}^{(0)}(t)=-i\left\langle 0\left|\mathcal{T} b_{0}(t) b_{0}^{\dagger}(0)\right| 0\right\rangle, \\
G_{a_{\mathbf{k}} b}^{(0)}(t) & =-i\left\langle 0\left|\mathcal{T} a_{\mathbf{k}}(t) b_{0}^{\dagger}(0)\right| 0\right\rangle,
\end{aligned}
$$

where $\mathcal{T}$ is the time-ordering operator. The Fourier transforms $G_{\alpha b}^{(0)}(\omega)=\int d t G_{\alpha b}^{(0)}(t) e^{i \omega t}\left(\alpha=a_{\mathbf{k}}, \quad b_{0}\right)$ can be obtained by integrating out the bath modes as

$$
\begin{gathered}
G_{a_{\mathbf{k}} b}^{(0)}(\omega)=\frac{\Omega \eta_{\mathbf{k}}}{\sqrt{V}} \frac{G_{b}^{(0)}(\omega)}{\omega-\varepsilon_{\mathbf{k}}+i 0^{+}}, \\
G_{b}^{(0)}(\omega)=\left[\omega-\Delta-\int \frac{d^{d} \mathbf{k}}{(2 \pi)^{d}} \frac{\Omega^{2} \eta_{\mathbf{k}}^{2}}{\omega-\varepsilon_{\mathbf{k}}+i 0^{+}}\right]^{-1} .
\end{gathered}
$$

The poles of $G_{b}^{(0)}(\omega)$ correspond to the bound state energies as shown in Eq. (B7).

In terms of the eigenstates and eigenvalues of $\mathcal{H}_{0}$, the Green function

$$
G_{b}^{(0)}(\omega)=\sum_{\lambda} \frac{\left|u_{\lambda}\right|^{2}}{\omega-E_{1 \lambda}+i 0^{+}}
$$

is constructed using the Lehmann representation.

\section{1. $N=2$ subspace}

In the $N=2$ subspace, the most general eigenstate with eigenenergy $E_{2 \lambda}$ has the form

$$
\begin{aligned}
\left|2_{\lambda}\right\rangle= & {\left[u_{\lambda}^{(2)} b_{0}^{\dagger 2}+\sum_{\mathbf{k}} f_{1 \lambda}^{(2)}(\mathbf{k}) a_{\mathbf{k}}^{\dagger} b_{0}^{\dagger}\right.} \\
& \left.+\sum_{\mathbf{k}_{1}, \mathbf{k}_{2}} f_{2 \lambda}^{(2)}\left(\mathbf{k}_{1}, \mathbf{k}_{2}\right) a_{\mathbf{k}_{1}}^{\dagger} a_{\mathbf{k}_{2}}^{\dagger}\right]|0\rangle .
\end{aligned}
$$

We now compute the bound state $(\lambda=B)$ energy $E_{2}$, the coefficient $u_{B}^{(2)}$, and the bath wave functions $f_{1 B}^{(2)}(\mathbf{k})$ and $f_{2 B}^{(2)}\left(\mathbf{k}_{1}, \mathbf{k}_{2}\right)$ using the Green function method. The twoparticle Green functions are defined as

$$
G_{\alpha_{1} \alpha_{2}}(t)=-i\left\langle 0\left|\mathcal{T} \alpha_{1}(t) \alpha_{2}(t) \alpha_{2}^{\dagger} \alpha_{1}^{\dagger}\right| 0\right\rangle,
$$

where there are three different possible choices: (i) $\alpha_{1}=$ $\alpha_{2}=b_{0}$, (ii) $\alpha_{1}=b_{0}, \alpha_{2}=a_{\mathbf{k}}$, or (iii) $\alpha_{1}=a_{\mathbf{k}_{2}}, \alpha_{2}=a_{\mathbf{k}_{1}}$. In the Lehmann representation, the Green function

$G_{\alpha_{1} \alpha_{2}}(\omega)=\int d t G_{\alpha_{1} \alpha_{2}}(t) e^{i \omega t}=\sum_{\lambda} \frac{\left\langle 0\left|\alpha_{1} \alpha_{2}\right| 2_{\lambda}\right\rangle\left\langle 2_{\lambda}\left|\alpha_{2}^{\dagger} \alpha_{1}^{\dagger}\right| 0\right\rangle}{\omega-E_{2 \lambda}+i 0^{+}}$,

whose poles and corresponding residues determine the bound state energies $E_{2}$ and wave functions $\left\langle 0\left|\alpha_{1} \alpha_{2}\right| B_{2}\right\rangle$ 
$\left[u_{B}^{(2)}=\left\langle 0\left|b_{0}^{2}\right| B_{2}\right\rangle / 2, \quad f_{1 B}^{(2)}(\mathbf{k})=\left\langle 0\left|b_{0} a_{\mathbf{k}}\right| B_{2}\right\rangle\right.$ and $f_{2 B}^{(2)}\left(\mathbf{k}_{1}\right.$, $\left.\left.\mathbf{k}_{2}\right)=\left\langle 0\left|a_{\mathbf{k}_{2}} a_{\mathbf{k}_{1}}\right| B_{2}\right\rangle / 2\right]$.

The Dyson expansion of the two-body interaction $H_{\mathrm{hc}}$ results in the connected part $\left[G_{\alpha_{1} \alpha_{2}}(\omega)\right]_{c}$ of the two-body Green function:

$$
\left[G_{\alpha_{1} \alpha_{2}}(\omega)\right]_{c}=2 \Pi_{\alpha_{1} \alpha_{2}}(\omega) T_{2}(\omega) \Pi_{\alpha_{1} \alpha_{2}}^{*}(\omega) .
$$

The poles of the $T$ matrix,

$$
T_{2}(\omega)=\frac{1}{U_{0}^{-1}-\Pi_{b b}(\omega)},
$$

give the bound state energies, where

$$
\Pi_{b b}(\omega)=\sum_{\lambda \lambda^{\prime}} \frac{\left|u_{\lambda}\right|^{2}\left|u_{\lambda^{\prime}}\right|^{2}}{\omega-E_{1 \lambda}-E_{1 \lambda^{\prime}}+i 0^{+}}
$$

can be computed from the bubble diagram. In the vicinity of a pole $E_{2}$, the $T$ matrix $T_{2}(\omega) \sim Z_{2 B} /\left(\omega-E_{2}+i 0^{+}\right)$ with a residue

$$
Z_{2 B}^{-1}=\sum_{\lambda \lambda^{\prime}} \frac{\left|u_{\lambda}\right|^{2}\left|u_{\lambda^{\prime}}\right|^{2}}{\left(E_{2}-E_{1 \lambda}-E_{1 \lambda^{\prime}}\right)^{2}} .
$$

The wave functions can be determind by $\Pi_{\alpha_{1} \alpha_{2}}(\omega)$, defined as

$$
\Pi_{b a_{\mathbf{k}}}(\omega)=\frac{\Omega \eta_{\mathbf{k}}}{\sqrt{V}} \sum_{\lambda} \frac{\left|u_{\lambda}\right|^{2} G_{b}^{(0)}\left(\omega-E_{1 \lambda}\right)}{\omega-\varepsilon_{\mathbf{k}}-E_{1 \lambda}+i 0^{+}}
$$

and

$$
\begin{aligned}
& \Pi_{a_{\mathbf{k}_{2}} a_{\mathbf{k}_{1}}}(\omega) \\
& =\frac{\Omega^{2}}{V} \eta_{\mathbf{k}_{1}} \eta_{\mathbf{k}_{2}} \sum_{\lambda \lambda^{\prime}} \frac{\left|u_{\lambda}\right|^{2}\left|u_{\lambda^{\prime}}\right|^{2}}{\left(\omega-\varepsilon_{\mathbf{k}_{1}}-\varepsilon_{\mathbf{k}_{2}}+i 0^{+}\right)\left(\omega-E_{1 \lambda}-E_{1 \lambda^{\prime}}+i 0^{+}\right)} \\
& \quad \times \frac{2 \omega-\varepsilon_{\mathbf{k}_{1}}-\varepsilon_{\mathbf{k}_{2}}-E_{1 \lambda}-E_{1 \lambda^{\prime}}}{\left(\omega-\varepsilon_{\mathbf{k}_{1}}-E_{1 \lambda}+i 0^{+}\right)\left(\omega-E_{1 \lambda^{\prime}}-\varepsilon_{\mathbf{k}_{2}}+i 0^{+}\right)} .
\end{aligned}
$$

The variables appearing in the wave functions are $u_{B}^{(2)}=\sqrt{Z_{2 B} / 2} \Pi_{b b}\left(E_{2}\right), f_{1 B}^{(2)}(\mathbf{k})=\sqrt{2 Z_{2 B}} \Pi_{b a_{\mathbf{k}}}\left(E_{2}\right)$, and $f_{2 B}^{(2)}\left(\mathbf{k}_{1}, \mathbf{k}_{2}\right)=\sqrt{Z_{2 B} / 2} \Pi_{a_{\mathbf{k}_{2}} a_{\mathbf{k}_{1}}}\left(E_{2}\right)$. The real-space wave functions obtained via Fourier transforms are

$$
\begin{gathered}
f_{1 B}^{(2)}\left(\mathbf{r}_{j}\right)=\frac{1}{\sqrt{V}} \sum_{\mathbf{k}} f_{1 B}^{(2)}(\mathbf{k}) e^{i \mathbf{k} \cdot \mathbf{r}_{j}}, \\
f_{2 B}^{(2)}\left(\mathbf{r}_{j_{1}}, \mathbf{r}_{j_{2}}\right)=\frac{1}{V} \sum_{\mathbf{k}_{1} \mathbf{k}_{2}} f_{2 B}^{(2)}\left(\mathbf{k}_{1}, \mathbf{k}_{2}\right) e^{i \mathbf{k}_{1} \cdot \mathbf{r}_{j_{1}}+i \mathbf{k}_{2} \cdot \mathbf{r}_{j_{2}}} .
\end{gathered}
$$

In the hard-core limit $U_{0} \rightarrow \infty, T_{2}^{-1}\left(E_{2}\right)=0$ gives $\Pi_{b}\left(E_{2}\right)=0$ and $u_{B}^{(2)}=0$, which means that there is no double occupation in the $b_{0}$ mode.

\section{2. $N=3$ subspace}

In the $N=3$ subspace, the most general eigenstate reads

$$
\begin{aligned}
\left|3_{\lambda}\right\rangle= & {\left[u_{\lambda}^{(3)} b_{0}^{\dagger 3}+\sum_{\mathbf{k}} f_{1 \lambda}^{(3)}(\mathbf{k}) a_{\mathbf{k}}^{\dagger} b_{0}^{\dagger 2}\right.} \\
& +\sum_{\mathbf{k}_{1} \mathbf{k}_{2}} f_{2 \lambda}^{(3)}\left(\mathbf{k}_{1}, \mathbf{k}_{2}\right) a_{\mathbf{k}_{1}}^{\dagger} a_{\mathbf{k}_{2}}^{\dagger} b_{0}^{\dagger} \\
& \left.+\sum_{\mathbf{k}_{1} \mathbf{k}_{2} \mathbf{k}_{3}} f_{3 \lambda}^{(3)}\left(\mathbf{k}_{1}, \mathbf{k}_{2}, \mathbf{k}_{3}\right) a_{\mathbf{k}_{1}}^{\dagger} a_{\mathbf{k}_{2}}^{\dagger} a_{\mathbf{k}_{3}}^{\dagger}\right]|0\rangle .
\end{aligned}
$$

The three-particle bound state can be studied by the threeparticle Green function

$$
G_{3}(t)=-i\left\langle\mathcal{T} \alpha_{3}(t) \alpha_{2}(t) \alpha_{1}(t) \alpha_{1}^{\dagger} \alpha_{2}^{\dagger} \alpha_{3}^{\dagger}\right\rangle,
$$

where the bound state energy $E_{3}$, the coefficient $u_{B}^{(3)}$, and the wave functions $f_{1 B}^{(3)}(\mathbf{k}), f_{2 B}^{(3)}\left(\mathbf{k}_{1}, \mathbf{k}_{2}\right)$, and $f_{3 B}^{(3)}\left(\mathbf{k}_{1}, \mathbf{k}_{2}, \mathbf{k}_{3}\right)$ can be obtained by four different choices of operators in $G_{3}(t)$ : (i) $\alpha_{1}=\alpha_{2}=\alpha_{3}=b_{0}$, (ii) $\alpha_{1}=a_{\mathbf{k}}$, $\alpha_{2}=\alpha_{3}=b_{0}$, (iii) $\alpha_{1}=a_{\mathbf{k}_{1}}, \alpha_{2}=a_{\mathbf{k}_{2}}, \alpha_{3}=b_{0}$, and (iv) $\alpha_{1}=a_{\mathbf{k}_{1}}, \alpha_{2}=a_{\mathbf{k}_{2}}, \alpha_{3}=a_{\mathbf{k}_{3}}$.

In the Lehmann representation, the Green function

$$
G_{3}(\omega)=\int d t G_{3}(t) e^{i \omega t}=\sum_{\lambda} \frac{\left\langle 0\left|\alpha_{3} \alpha_{2} \alpha_{1}\right| 3_{\lambda}\right\rangle\left\langle 3_{\lambda}\left|\alpha_{1}^{\dagger} \alpha_{2}^{\dagger} \alpha_{3}^{\dagger}\right| 0\right\rangle}{\omega-E_{3 \lambda}+i 0^{+}},
$$

whose poles and corresponding residues determine the bound state energies $E_{3}$ and the wave functions $\left\langle 0\left|\alpha_{3} \alpha_{2} \alpha_{1}\right| B_{3}\right\rangle$ $\left[u_{B}^{(3)}=\left\langle 0\left|b_{0}^{3}\right| 3_{\lambda}\right\rangle / 6, \quad f_{1 B}^{(3)}(\mathbf{k})=\left\langle 0\left|b_{0}^{2} a_{\mathbf{k}}\right| B_{3}\right\rangle / 2, \quad f_{2 B}^{(3)}\left(\mathbf{k}_{1}, \mathbf{k}_{2}\right)=\left\langle 0\left|b_{0} a_{\mathbf{k}_{2}} a_{\mathbf{k}_{1}}\right| B_{3}\right\rangle / 2, \quad\right.$ and $\quad f_{3 \lambda}^{(3)}\left(\mathbf{k}_{1}, \mathbf{k}_{2}, \mathbf{k}_{3}\right)=$ $\left.\left\langle 0\left|a_{\mathbf{k}_{3}} a_{\mathbf{k}_{2}} a_{\mathbf{k}_{1}}\right| B_{3}\right\rangle / 6\right]$.

The Dyson expansion gives to the connected part

$$
\left[G_{3}(\omega)\right]_{c}=\int \frac{d \omega_{1} d \omega_{1}^{\prime}}{(2 \pi)^{2}} \chi_{3}\left(\omega, \omega_{1}\right) T_{3}\left(\omega, \omega_{1}, \omega_{1}^{\prime}\right) \chi_{3}\left(\omega, \omega_{1}^{\prime}\right)
$$

where 


$$
\chi_{3}\left(\omega, \omega_{1}\right)=P_{123}\left[G_{\alpha_{i_{1}} b}^{(0)}\left(\omega_{1}\right) T_{2}\left(\omega-\omega_{1}\right) \int \frac{d \omega_{2}}{2 \pi} G_{\alpha_{i_{2}} b}^{(0)}\left(\omega_{2}\right) G_{\alpha_{i_{3}} b}^{(0)}\left(\omega-\omega_{1}-\omega_{2}\right)\right]
$$

is determined by the operator $P_{123}$ which permutes $\alpha_{1,2,3}$. The three-particle $T$ matrix satisfies the integral equation

$$
T_{3}\left(\omega, \omega_{1}, \omega_{1}^{\prime}\right)=G_{b}^{(0)}\left(\omega-\omega_{1}-\omega_{1}^{\prime}\right)+2 i \int \frac{d \omega^{\prime}}{2 \pi} G_{b}^{(0)}\left(\omega-\omega_{1}-\omega^{\prime}\right) T_{2}\left(\omega-\omega^{\prime}\right) G_{b}^{(0)}\left(\omega^{\prime}\right) T_{3}\left(\omega, \omega^{\prime}, \omega_{1}^{\prime}\right) .
$$

In the vicinity of the pole $E_{3}$, the three-particle $T$ matrix has the form

$$
T_{3}\left(\omega, \omega_{1}, \omega_{1}^{\prime}\right)=\frac{F\left(\omega_{1}\right) F\left(\omega_{1}^{\prime}\right)}{\omega-E_{3}+i 0^{+}},
$$

where $F(\omega)$ is determined by the integral equation

$$
F(\omega)=2 i \int \frac{d \omega^{\prime}}{2 \pi} G_{b}^{(0)}\left(E_{3}-\omega-\omega^{\prime}\right) T_{2}\left(E_{3}-\omega^{\prime}\right) G_{b}^{(0)}\left(\omega^{\prime}\right) F\left(\omega^{\prime}\right)
$$

Using the analyticity of $F(\omega)$ and the residue theorem, we find that the integral in Eq. (B31) becomes

$$
F(\omega)=2 \sum_{\lambda}\left|u_{\lambda}\right|^{2} G_{b}^{(0)}\left(E_{3}-\omega-E_{1 \lambda}\right) T_{2}\left(E_{3}-E_{1 \lambda}\right) F\left(E_{1 \lambda}\right) .
$$

For the on-shell frequency $\omega=E_{1 \lambda}$, we obtain the matrix equation

$$
\sum_{\lambda^{\prime}} M_{\lambda \lambda^{\prime}} F\left(E_{1 \lambda^{\prime}}\right)=0
$$

where

$$
M_{\lambda \lambda^{\prime}}=2\left|u_{\lambda^{\prime}}\right|^{2} G_{b}^{(0)}\left(E_{3}-E_{1 \lambda}-E_{1 \lambda^{\prime}}\right) T_{2}\left(E_{3}-E_{1 \lambda^{\prime}}\right)-\delta_{\lambda \lambda^{\prime}}
$$

The bound state energy $E_{3}$ is obtained by solving the equation $\operatorname{det} M=0$. The eigenvector $F\left(E_{1 \lambda}\right)$ with zero eigenvalue can be used to obtain the function $F(\omega)$ via Eq. (B31).

In the hard-core limit $U_{0} \rightarrow \infty$, the wave functions $u_{B}^{(3)}=f_{1 B}^{(3)}(\mathbf{k})=0$ vanish, and the residue of $G_{3}(\omega)$ gives the wave functions

$$
\begin{aligned}
f_{2 B}^{(3)}\left(\mathbf{k}_{1}, \mathbf{k}_{2}\right)= & -\frac{\Omega^{2}}{V} \eta_{\mathbf{k}_{1}} \eta_{\mathbf{k}_{2}} P_{12}\left\{\sum _ { \lambda _ { 1 } \lambda _ { 2 } \lambda _ { 3 } } \frac { | u _ { \lambda _ { 1 } } | ^ { 2 } | u _ { \lambda _ { 2 } } | ^ { 2 } | u _ { \lambda _ { 3 } } | ^ { 2 } } { 2 ( \varepsilon _ { \mathbf { k } _ { 1 } } - E _ { 1 \lambda _ { 1 } } ) } \left[\frac{T_{2}\left(E_{3}-E_{1 \lambda_{2}}\right) F\left(E_{1 \lambda_{2}}\right)+2 T_{2}\left(E_{3}-\varepsilon_{\mathbf{k}_{1}}\right) F\left(\varepsilon_{\mathbf{k}_{1}}\right)}{\left(E_{3}-\varepsilon_{\mathbf{k}_{1}}-\varepsilon_{\mathbf{k}_{2}}-E_{1 \lambda_{2}}\right)\left(E_{3}-\varepsilon_{\mathbf{k}_{1}}-E_{1 \lambda_{2}}-E_{1 \lambda_{3}}\right)}\right.\right. \\
& \left.\left.-\frac{T_{2}\left(E_{3}-E_{1 \lambda_{2}}\right) F\left(E_{1 \lambda_{2}}\right)+2 T_{2}\left(E_{3}-E_{1 \lambda_{1}}\right) F\left(E_{1 \lambda_{1}}\right)}{\left(E_{3}-\varepsilon_{\mathbf{k}_{2}}-E_{1 \lambda_{1}}-E_{1 \lambda_{2}}\right)\left(E_{3}-E_{1 \lambda_{1}}-E_{1 \lambda_{2}}-E_{1 \lambda_{3}}\right)}\right]\right\}
\end{aligned}
$$

and

$$
\begin{aligned}
f_{3 B}^{(3)}\left(\mathbf{k}_{1}, \mathbf{k}_{2}, \mathbf{k}_{3}\right)= & -\frac{\Omega^{3}}{6 V^{3 / 2}} \eta_{\mathbf{k}_{1}} \eta_{\mathbf{k}_{2}} \eta_{\mathbf{k}_{3}} P_{123}\left\{\sum _ { \lambda _ { 1 } \lambda _ { 2 } \lambda _ { 3 } } \frac { | u _ { \lambda _ { 1 } } | ^ { 2 } | u _ { \lambda _ { 2 } } | ^ { 2 } | u _ { \lambda _ { 3 } } | ^ { 2 } } { \varepsilon _ { \mathbf { k } _ { 1 } } - E _ { 1 \lambda _ { 1 } } } \left[\frac{T_{2}\left(E_{3}-\varepsilon_{\mathbf{k}_{1}}\right) F\left(\varepsilon_{\mathbf{k}_{1}}\right)}{\left(E_{3}-\varepsilon_{\mathbf{k}_{1}}-\varepsilon_{\mathbf{k}_{2}}-\varepsilon_{\mathbf{k}_{3}}\right)\left(E_{3}-\varepsilon_{\mathbf{k}_{1}}-\varepsilon_{\mathbf{k}_{2}}-E_{1 \lambda_{3}}\right)}\right.\right. \\
& \times \frac{2 E_{3}-2 \varepsilon_{\mathbf{k}_{1}}-\varepsilon_{\mathbf{k}_{2}}-\varepsilon_{\mathbf{k}_{3}}-E_{1 \lambda_{2}}-E_{1 \lambda_{3}}}{\left(E_{3}-\varepsilon_{\mathbf{k}_{1}}-\varepsilon_{\mathbf{k}_{3}}-E_{1 \lambda_{2}}\right)\left(E_{3}-\varepsilon_{\mathbf{k}_{1}}-E_{1 \lambda_{2}}-E_{1 \lambda_{3}}\right)}-\frac{T_{2}\left(E_{3}-E_{1 \lambda_{1}}\right) F\left(E_{1 \lambda_{1}}\right)}{\left(E_{3}-\varepsilon_{\mathbf{k}_{2}}-\varepsilon_{\mathbf{k}_{3}}-E_{1 \lambda_{1}}\right)\left(E_{3}-\varepsilon_{\mathbf{k}_{2}}-E_{1 \lambda_{1}}-E_{1 \lambda_{3}}\right)} \\
& \left.\left.\times \frac{2 E_{3}-\varepsilon_{\mathbf{k}_{2}}-\varepsilon_{\mathbf{k}_{3}}-2 E_{1 \lambda_{1}}-E_{1 \lambda_{2}}-E_{1 \lambda_{3}}}{\left(E_{3}-\varepsilon_{\mathbf{k}_{3}}-E_{1 \lambda_{1}}-E_{1 \lambda_{2}}\right)\left(E_{3}-E_{1 \lambda_{1}}-E_{1 \lambda_{2}}-E_{1 \lambda_{3}}\right)}\right]\right\}
\end{aligned}
$$

where $P_{12}$ and $P_{13}$ are permutations for $\mathbf{k}_{1}, \mathbf{k}_{2}$ and $\mathbf{k}_{1}, \mathbf{k}_{2}, \mathbf{k}_{3}$, respectively. The real-space wave functions can be obtained via Fourier transforms 


$$
\begin{aligned}
f_{2 B}^{(3)}\left(\mathbf{r}_{j_{1}}, \mathbf{r}_{j_{2}}\right) & =\frac{1}{V} \sum_{\mathbf{k}_{1} \mathbf{k}_{2}} f_{2 B}^{(3)}\left(\mathbf{k}_{1}, \mathbf{k}_{2}\right) e^{i \mathbf{k}_{1} \cdot \mathbf{r}_{j_{1}}+i \mathbf{k}_{2} \cdot \mathbf{r}_{j_{2}}} \\
f_{3 B}^{(3)}\left(\mathbf{r}_{j_{1}}, \mathbf{r}_{j_{2}}, \mathbf{r}_{j_{3}}\right) & =\frac{1}{V^{3 / 2}} \sum_{\mathbf{k}_{1} \mathbf{k}_{2} \mathbf{k}_{3}} f_{3 B}^{(3)}\left(\mathbf{k}_{1}, \mathbf{k}_{2}, \mathbf{k}_{3}\right) e^{i \mathbf{k}_{1} \cdot \mathbf{r}_{j_{1}}+i \mathbf{k}_{2} \cdot \mathbf{r}_{j_{2}}+i \mathbf{k}_{3} \cdot \mathbf{r}_{j_{3}}} .
\end{aligned}
$$

\section{APPENDIX C: VARIATIONAL ANSATZ}

This appendix provides full details about the optimization process of the variational Ansatz.

By a direct computation, the energy of the ground-state variational Ansatz Eq. (13) is

$$
E_{\text {g.s. }}=\left\langle B_{N}|H| B_{N}\right\rangle=(N-1) h_{A A}+v^{T} \mathcal{E}_{0} v,
$$

where $\mathcal{E}_{0}$ is a matrix (see below), $h_{A A}$ is one of its elements, and $v=(\alpha, \beta, \gamma)^{T}$ satisfies the normalization condition $v^{T} v=1$. We introduce variables $h_{M M^{\prime}}=$ $\sum_{\mathbf{k}} \varepsilon_{\mathbf{k}} \varphi_{M}(\mathbf{k}) \varphi_{M^{\prime}}(\mathbf{k}) \quad$ and $\quad I_{M}=\sum_{\mathbf{k}} \eta_{\mathbf{k}} \varphi_{M}(\mathbf{k}) / \sqrt{V}$ $\left(M, M^{\prime}=A, B\right)$ to define the matrix

$$
\mathcal{E}_{0}=\left(\begin{array}{ccc}
\Delta & \sqrt{N} \Omega I_{A} & \Omega I_{B} \\
\sqrt{N} \Omega I_{A} & h_{A A} & \sqrt{N} h_{A B} \\
\Omega I_{B} & \sqrt{N} h_{A B} & h_{B B}
\end{array}\right) .
$$

The orthonormal collective modes we introduce in the main text satisfy the constraints $\left[A, A^{\dagger}\right]=\left[B, B^{\dagger}\right]=1$ and $\left[A, B^{\dagger}\right]=0$, so we have

$$
\sum_{\mathbf{k}} \varphi_{M}^{2}(\mathbf{k})=1 \quad \text { and } \quad \sum_{\mathbf{k}} \varphi_{A}(\mathbf{k}) \varphi_{B}(\mathbf{k})=0
$$

To optimize the ground-state energy under these constraints, we introduce Lagrangian multipliers $\lambda, \mu_{A}, \mu_{B}$, and $\mu_{A B}$ to define the function

$$
\begin{aligned}
F_{\text {g.s. }}= & E_{\text {g.s. }}-\mu_{A} \sum_{\mathbf{k}} \varphi_{A}^{2}(\mathbf{k})-\mu_{B} \sum_{\mathbf{k}} \varphi_{B}^{2}(\mathbf{k}) \\
& -2 \mu_{A B} \sum_{\mathbf{k}} \varphi_{A}(\mathbf{k}) \varphi_{B}(\mathbf{k})-\lambda\left(\alpha^{2}+\beta^{2}+\gamma^{2}\right) .
\end{aligned}
$$

By taking derivatives with respect to $v$ and $\varphi_{M}(\mathbf{k})$, we obtain a set of coupled nonlinear Gross-Pitaevski-like equations:

$$
\mathcal{E}_{0} v=\lambda v
$$

and

$$
\mathcal{H}_{\mathrm{GP}}\left(\begin{array}{c}
\varphi_{A}(\mathbf{k}) \\
\varphi_{B}(\mathbf{k})
\end{array}\right)+\frac{\Omega \eta_{\mathbf{k}}}{\sqrt{V}} \alpha\left(\begin{array}{c}
\sqrt{N} \beta \\
\gamma
\end{array}\right)=\mu\left(\begin{array}{c}
\varphi_{A}(\mathbf{k}) \\
\varphi_{B}(\mathbf{k})
\end{array}\right),
$$

where

$$
\mathcal{H}_{\mathrm{GP}}=\left(\begin{array}{cc}
\left(N-1+\beta^{2}\right) \varepsilon_{\mathbf{k}} & \beta \gamma \sqrt{N} \varepsilon_{\mathbf{k}}-\mu_{A B} \\
\beta \gamma \sqrt{N} \varepsilon_{\mathbf{k}}-\mu_{A B} & \gamma^{2} \varepsilon_{\mathbf{k}}
\end{array}\right) .
$$

The "chemical potentials" $\mu=\mu_{A}=\mu_{B}$, and $\mu_{A B}=$ $\beta \gamma \sqrt{N} h_{A A}+\gamma^{2} h_{A B}+\Omega \alpha \gamma I_{A}$ due to the constraint $\sum_{\mathbf{k}} \varphi_{A}(\mathbf{k}) \varphi_{B}(\mathbf{k})=0$. The solutions $\varphi_{M}(\mathbf{k})$ to these equations are

$$
\varphi_{M}(\mathbf{k})=\frac{1}{\sqrt{V}} \sum_{\mu=1,2} \frac{c_{M, \mu}}{\sqrt{\mathcal{N}_{\mu \mu}}} \frac{\eta_{\mathbf{k}}}{e_{\mu}-\varepsilon_{\mathbf{k}}},
$$

where we introduce variables $c_{M, \mu}, e_{\mu}$, and

$$
\mathcal{N}_{\mu \nu}=\int \frac{d^{d} \mathbf{k}}{(2 \pi)^{d}} \frac{\eta_{\mathbf{k}}^{2}}{\left(e_{\mu}-\varepsilon_{\mathbf{k}}\right)\left(e_{\nu}-\varepsilon_{\mathbf{k}}\right)} .
$$

The two coefficients $c_{M, \mu}$ are related as $c_{M, 2}=t_{M} c_{M, 1}$. The constraints given by Eq. (C3) result in the relations

$$
\begin{aligned}
c_{M, 1} & =\frac{1}{\sqrt{1+t_{M}^{2}+2 \frac{\mathcal{N}_{12}}{\sqrt{\mathcal{N}_{11} \mathcal{N}_{22}}} t_{M}}}, \\
t_{B} & =-\frac{\sqrt{\mathcal{N}_{11} \mathcal{N}_{22}}+t_{A} \mathcal{N}_{12}}{\sqrt{\mathcal{N}_{11} \mathcal{N}_{22}} t_{A}+\mathcal{N}_{12}} .
\end{aligned}
$$

To find optimized variational parameters, we construct the wave functions $\varphi_{M}(\mathbf{k})$ in Eq. (C8) and the matrix $\mathcal{E}_{0}$ in Eq. (C2) using different sets of $e_{1}, e_{2}$, and $t_{A}$. For a given set of $e_{1}, e_{2}$, and $t_{A}$, the ground-state energy is given by $E_{\text {g.s. }}=(N-1) h_{A A}+\lambda_{0}$, where $\lambda_{0}$ is the lowest eigenvalue of $\mathcal{E}_{0}$. The optimized $e_{1}, e_{2}$, and $t_{A}$ are those that give the lowest $E_{\text {g.s. }}$. To reveal the properties of the MEBS in coordinate space, we can Fourier transform the momentum space mode functions $\varphi_{M}(\mathbf{k})$ into real space to obtain $M^{\dagger}=\sum_{j} \varphi_{M, j} a_{j}^{\dagger}$, with

$$
\varphi_{M, j}=\sum_{\mu=1,2} c_{M, \mu} \sqrt{\frac{1-x_{\mu}^{2}}{1+x_{\mu}^{2}}} x_{\mu}^{|j|},
$$

where $x_{\mu}+x_{\mu}^{-1}=2-e_{\mu}$, and $-1 / \log \left(x_{\mu}\right)$ is the localization length. 
[1] S. John and J. Wang, Quantum Electrodynamics near a Photonic Band Gap: Photon Bound States and Dressed Atoms, Phys. Rev. Lett. 64, 2418 (1990).

[2] V. P. Bykov, Spontaneous Emission from a Medium with a Band Spectrum, Sov. J. Quantum Electron. 4, 861 (1975).

[3] G. Kurizki, Two-Atom Resonant Radiative Coupling in Photonic Band Structures, Phys. Rev. A 42, 2915 (1990).

[4] V. Efimov, Energy Levels Arising from Resonant Two-Body Forces in a Three-Body System, Phys. Lett. B 33, 563 (1970).

[5] J. Bardeen, L. N. Cooper, and J. R. Schrieffer, Theory of Superconductivity, Phys. Rev. 108, 1175 (1957).

[6] T. Holstein, Studies of Polaron Motion: Part I. The Molecular-Crystal Model, Ann. Phys. (N.Y.) 8, 325 (1959).

[7] T. Holstein, Studies of Polaron Motion: Part II. The Small Polaron, Ann. Phys. (N.Y.) 8, 343 (1959).

[8] A. Goban et al., Atom-Light Interactions in Photonic Crystals, Nat. Commun. 5, 3808 (2014).

[9] S.-P. Yu, J. D. Hood, J. A. Muniz, M. J. Martin, R. Norte, C.-L. Hung, S. M. Meenehan, J. D. Cohen, O. Painter, and H. J. Kimble, Nanowire Photonic Crystal Waveguides for Single-Atom Trapping and Strong Light-Matter Interactions, Appl. Phys. Lett. 104, 111103 (2014).

[10] P. Lodahl, S. Mahmoodian, and S. Stobbe, Interfacing Single Photons and Single Quantum Dots with Photonic Nanostructures, Rev. Mod. Phys. 87, 347 (2015).

[11] A. Laucht et al., A Waveguide-Coupled On-Chip SinglePhoton Source, Phys. Rev. X 2, 011014 (2012).

[12] M. Arcari et al., Near-Unity Coupling Efficiency of a Quantum Emitter to a Photonic Crystal Waveguide, Phys. Rev. Lett. 113, 093603 (2014).

[13] J. D. Thompson, T. G. Tiecke, N. P. de Leon, J. Feist, A. V. Akimov, M. Gullans, A. S. Zibrov, V. Vuletic, and M. D. Lukin, Coupling a Single Trapped Atom to a Nanoscale Optical Cavity, Science 340, 1202 (2013).

[14] E. Shahmoon and G. Kurizki, Nonradiative Interaction and Entanglement between Distant Atoms, Phys. Rev. A 87, 033831 (2013).

[15] J. S. Douglas, H. Habibian, C.-L. Hung, A. V. Gorshkov, H. J. Kimble, and D.E. Chang, Quantum Many-Body Models with Cold Atoms Coupled to Photonic Crystals, Nat. Photonics 9, 326 (2015).

[16] A. González-Tudela, C.-L. Hung, D. Chang, J. Cirac, and H. Kimble, Subwavelength Vacuum Lattices and AtomAtom Interactions in Two-Dimensional Photonic Crystals, Nat. Photonics 9, 320 (2015).

[17] P. Longo, P. Schmitteckert, and K. Busch, Few-Photon Transport in Low-Dimensional Systems: InteractionInduced Radiation Trapping, Phys. Rev. Lett. 104, 023602 (2010).

[18] T. Shi and C. P. Sun, Lehmann-Symanzik-Zimmermann Reduction Approach to Multiphoton Scattering in Coupled-Resonator Arrays, Phys. Rev. B 79, 205111 (2009).

[19] T. Shi and C. Sun, Two-Photon Scattering in One Dimension by Localized Two-Level System, arXiv:0907.2776.
[20] C.-z. Zhu, S. Endo, P. Naidon, and P. Zhang, Scattering and Bound States of Two Polaritons in an Array of Coupled Cavities, Few-Body Syst. 54, 1921 (2013).

[21] Ş. E. Kocabaş, Effects of Modal Dispersion on Few PhotonQubit Scattering in One-Dimensional Waveguides, Phys. Rev. A 93, 033829 (2016).

[22] M. P. Schneider, T. Sproll, C. Stawiarski, P. Schmitteckert, and K. Busch, Green's Function Formalism for Waveguide QED Applications, Phys. Rev. A 93, 013828 (2016).

[23] E. Sanchez-Burillo, D. Zueco, J. J. Garcia-Ripoll, and L. Martin-Moreno, Scattering in the Ultrastrong Regime: Nonlinear Optics with One Photon, Phys. Rev. Lett. 113, 263604 (2014).

[24] G. Díaz-Camacho, A. Bermudez, and J. J. García-Ripoll, Dynamical Polaron Ansatz: A Theoretical Tool for the Ultrastrong-Coupling Regime of Circuit QED, Phys. Rev. A 93, 043843 (2016).

[25] S. R. White, Density Matrix Formulation for Quantum Renormalization Groups, Phys. Rev. Lett. 69, 2863 (1992).

[26] U. Schollwöck, The Density-Matrix Renormalization Group in the Age of Matrix Product States, Ann. Phys. (Amsterdam) 326, 96 (2011).

[27] J. Mlynek, A. Abdumalikov, C. Eichler, and A. Wallraff, Observation of Dicke Superradiance for Two Artificial Atoms in a Cavity with High Decay Rate, Nat. Commun. 5, 5186 (2014).

[28] I. de Vega, D. Porras, and J. Ignacio Cirac, Matter-Wave Emission in Optical Lattices: Single Particle and Collective Effects, Phys. Rev. Lett. 101, 260404 (2008).

[29] C. Navarrete-Benlloch, I. de Vega, D. Porras, and J. I. Cirac, Simulating Quantum-Optical Phenomena with Cold Atoms in Optical Lattices, New J. Phys. 13, 023024 (2011).

[30] W. L. Yang, J.-H. An, C. Zhang, M. Feng, and C. H. Oh, Preservation of Quantum Correlation between Separated Nitrogen-Vacancy Centers Embedded in Photonic-Crystal Cavities, Phys. Rev. A 87, 022312 (2013).

[31] L. Zhou, Z. R. Gong, Y.-x. Liu, C. P. Sun, and F. Nori, Controllable Scattering of a Single Photon inside a OneDimensional Resonator Waveguide, Phys. Rev. Lett. 101, 100501 (2008).

[32] E. Jaynes and F. Cummings, Comparison of Quantum and Semiclassical Radiation Theory with Application to the Beam Maser, Proc. IEEE 51, 89 (1963).

[33] F. Scazza, C. Hofrichter, M. Höfer, P. C. De Groot, I. Bloch, and S. Fölling, Observation of Two-Orbital Spin-Exchange Interactions with Ultracold Su (N)-Symmetric Fermions, Nat. Phys. 10, 779 (2014).

[34] M. Höfer, L. Riegger, F. Scazza, C. Hofrichter, D. R. Fernandes, M. M. Parish, J. Levinsen, I. Bloch, and S. Fölling, Observation of an Orbital Interaction-Induced Feshbach Resonance in ${ }^{173} \mathrm{Yb}$, Phys. Rev. Lett. 115, 265302 (2015).

[35] G. Calajó, F. Ciccarello, D. Chang, and P. Rabl, Atom-Field Dressed States in Slow-Light Waveguide QED, Phys. Rev. A 93, 033833 (2016). 